\title{
Enhanced Visual Evaluation of Feature Extractors for Image Mining
}

\author{
José Fernando Rodrigues Jr., Agma J. M. Traina, Caetano Traina Jr. \\ Computer Science Department \\ University of Sao Paulo at Sao Carlos - Brazil \\ Phone: +55-16-273-9674 \\ e-mail: [junio |agma | caetano]@icmc.usp.br \\ IEEE Copyright - http://ieeexplore.ieee.org/stamp/stamp.jsp?tp=\&arnumber=1387042
}

\begin{abstract}
This paper introduces a novel approach to evaluate, timely and effectively, the suitability of new image feature extraction techniques concerning similarity queries using CBIR systems. The proposed approach is based on two measurements derived from spatial properties intuitively and naturally perceived in spatial domains, and that can also be verified in multidimensional spaces. To bear out our proposal, we will show that the insights obtained by the proposed measurements comply with the well-known analysis methods based on the precision and recall approach.
\end{abstract}

\section{Introduction}

The management of large image databases, generated by nowadays computational systems, has motivated the database and image processing communities to research new algorithms to index and retrieve such large data items in a timely manner. As the amount of images generated adds to giga bytes per day in centers such as medical facilities, the indexing and retrieval should be as automated as possible. Therefore, this has motivated the development of contentbased image retrieval (CBIR) techniques, what is one of the keystones of the image mining techniques $[14,16]$.

The development of CBIR systems depends on a suitable definition of the image type in the database as well as on precise characteristics (features) extracted from the images. The features should represent their "essence", regarding a specific aspect of the images, in such a way that the images would be managed - indexed, compared and searched based on their features. However, extracting relevant features from images is not a trivial process, as such extraction processes should be done automatically due to the large volume of stored data. The features obtained by general extractors, the so-called low-level [17] features, usually presents a very low individual meaning, so it is common the existence of large semantic gaps between the extracted features and the high-level, semantic, expectation from the users when they perform queries.

Evaluating the usefulness of a new image feature extraction algorithm is an arduous task. Usually, it is done following a statistical approach, where it first needs to classify the image data set and then to apply many similarity queries over the data. Next, it is necessary to ask a human specialist on the domain to validate the answer given by the image retrieval technique supported by the extracted features [17]. Graphs of precision and recall [3] can be drawn according to the results given by the automatic approach based on the image features, allowing the comparison of the results obtained by the automatic extractors with those pointed out by the specialist. However, this approach is time consuming and can only be performed using small sets of images, because humans get tired fast during the analysis of images and the classification may result incorrect. Even worse, it can be skewed by the ability (or inability) of the analyst.

The objective of this paper is to create a technique to compare feature extractors and/or distance functions to answer the following questions:

1. Is a feature extractor/distance function adequate to discriminate images in a given domain?

2. Are the given feature extractors/distance functions better than another one for the full set of images, or are there classes in the image domain where the other extractor/distance perform better?

3. If different feature extractors/distance functions are suitable for different classes, which one is the most adequate for each class?

4. Is it possible to give clues to the feature extractors/distance functions developer so he/she can improve/fine tune the algorithms?

To answer such questions, we have developed a new technique that evaluates the applicability of image feature extractors/distance functions, measuring two properties derived from the numerical feature vectors extracted from the images. As detailed further, this approach analyzes the spatial distribution of the data items according to the feature vectors.

The remaining of this paper is structured as follows. 
Section 2 reviews the main concepts involved in this work, section 3 presents the feature extractors used in our experiments and section 4 presents the proposed properties and respective measurements. Section 5 shows the experiments performed in order to evaluate the usefulness of them and section 6 presents the conclusions.

\section{Background}

\subsection{Features spaces}

The first and crucial step in CBIR is the choice of the features to be extracted from the images in order to concisely represent the original image regarding a given criterion. Therefore, the extracted features are used in place of the images themselves, for indexing and retrieval, reducing the complexity of the comparisons needed for such operations. It is common that the features are organized in arrays, creating the feature vectors. They are employed to compare and generate index structures for managing images, using similarities that embody the visual differences between pairs of items [20]. Thus, access methods should be able to exploit the feature vectors, allowing efficient retrieval when answering similarity queries.

The most common and generic properties extracted from images are color, shape and texture, the so-called low-level features [15]. It is important to notice that, depending on the purpose of the query, different features would be used from the same data, and different ways of comparisons (distance functions) would be used to improve the retrieval process [13].

As the volume of data in image databases increases, it is important to look for reliable descriptions of the images that can be obtained automatically. There are basically two main approaches for that, extraction of features directly from the raw data, and extraction of features from transformed data. An example of features got from raw data is the count of pixels for each image color, which is given by the color histograms. Transformed data usually comes from a compressed transformation domain, such as wavelet coefficients [2].

\subsection{Similarity queries}

Given an image dataset $S$ represented by the feature vectors $s_{i}$ of each image, that is, $s_{i} \in S$, a similarity query always refers to a reference image called the query center. To answer a similarity query, the feature vector $s_{q}$ of the query center image is also extracted. For medical diagnosis based on images, it is helpful to retrieve cases that are similar to a given image under analysis. So, in this paper, we considered two classes of similarity queries, the range and the $k$-nearest neighbor queries.
Definition 1 (Nearest Neighbor query): Given a query center $s_{q}$ and the set of images $S$, the nearest neighbor is the unitary subset of $S$ such that $\operatorname{NNquery}\left(s_{q}\right)=\left\{s_{n} \in S \mid \forall\right.$ $\left.s_{i} \in S: d\left(s_{n}, s_{q}\right) \leq d\left(s_{i}, s_{q}\right)\right\}$.

It is frequently needed to get not only the nearest but a specific number $k$ of objects that are the nearest to the query center, represented by the $k$-NN query. For example, considering $k=5$, a nearest neighbor query on an image database domain should ask to "find in the given data set, the 5 images most similar to image A".

Definition 2 (Range query): Given a query center $s_{q}$, the set of images $S$, and a maximum search distance $r_{q}$, then Range query Rquery $\left(s_{q}, r_{q}\right)=\left\{s_{\mathrm{i}} \mid s_{\mathrm{i}} \in S: d\left(s_{i}, s_{q}\right) \leq r_{q}\right\}$.

An example of a range query is: "find every image that is within 10 units of distance from image A".

\subsection{Precision and recall}

The precision and recall is an approach that has been widely used to measure the retrieval effectiveness of an extraction technique $[1,3]$. Recall indicates the proportion of relevant images in the database which have been retrieved when answering a query. Precision is the proportion of the retrieved images that are relevant for the query.

Formally, let $X$ be the set of relevant images to answer a given query, let $Y$ be the set of retrieved images and $x, y$ and $z$ be respectively the number of images in the sets $X \cap Y$, $\bar{X} \cap Y$ and $X \cap \bar{Y}$. Thus, precision and recall can be expressed through the following conditional probabilities.

precision $=P(X \mid Y)=\frac{P(X \cap Y)}{P(Y)}=\frac{x}{x+y}$.
recall $=P(Y \mid X)=\frac{P(Y \cap X)}{P(X)}=\frac{x}{x+z}$

Ideally, the set of objects retrieved should be equal to the set of objects relevant for a specific query. However, mainly due to limitations on working with the features extracted from the images, as well as with the distance function used to compare them, this does not happen. Nevertheless, the measurement of precision versus recall is a valuable tool to evaluate the adequacy of distance functions and feature extractors. As a rule of thumb a precision versus recall graph close to the top indicates the best retrieval ability of the algorithm evaluated.

\subsection{Visualization applications}

Visualization has become an important tool in exploring, analyzing and understanding the information stored in databases. Appropriate information visualization tools often lead scientists to new insights about the data [10]. Visualization techniques are capable of revealing trends and 
patterns hidden in the data, as well as clusters, outliers, correlations and inconsistencies, providing a deep insight about the data distribution and behavior.

Several information visualization approaches have been presented in the literature to achieve the aforementioned objectives, including those based on hierarchical presentation [6], graph-based [4], pixel-oriented [9], geometric projection [7,8] and combinations of them [21]. For human beings, it is natural to understand objects distributed in a tridimensional Euclidean space. Therefore, in this work we looked for a technique that would map abstract information (multidimensional or even nondimensional data) into tridimensional space. This is achieved by the FastMap algorithm [5], which was originally proposed to reduce the dimensionality of a data set.

The measurements we propose are (hyper) space concerned, therefore, as will be seen, a spatial visualization can improve the comprehension promoted by such measurements.

\subsection{The FastMap technique and the FastMapDB tool}

The FastMap algorithm [5] was developed based on a remarkable improvement on the Multi Dimensional Scaling (MDS)[19], as the complexity of the FastMap is linear instead of the quadratic one for the MDS. The FastMap can be used to reduce the data dimensionality from an $n$-dimensional space to a $k$-dimensional one, where $k<n$. The sole information needed by the FastMap are the objects and a dissimilarity function, or distance function defined on

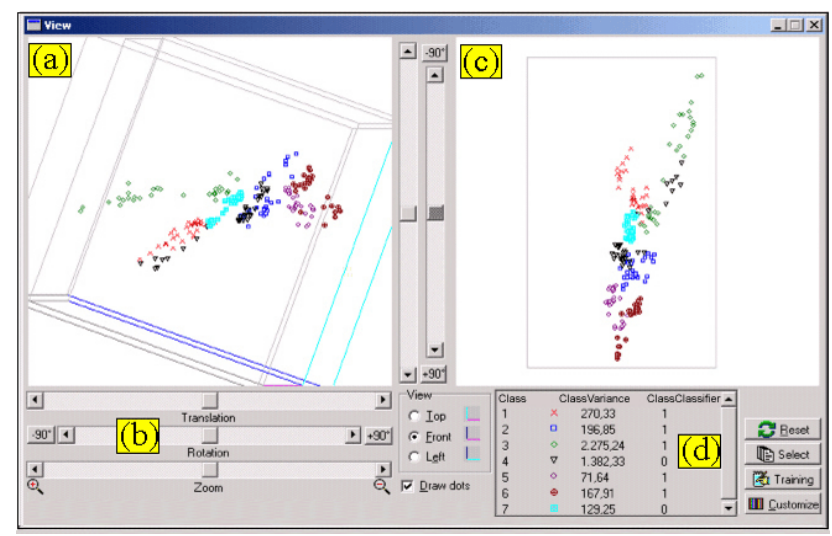

Figure 1. Visualization of the features DEnpropy (detailed in Section 4) extracted from the test image database. (a) The 3D presentation generated by the FastMapDB tool. (b) Slide buttons to interact with the 3D presentation. (c) A 2D projection over the front side of the 3D visualization. (d) The results of the measurements ClassVariance and ClassClassifier (section 4.1) for this data set. The seven colors and shapes of the points correspond to the seven distinct classes of images the data domain of the objects.

When the dimensional reduction is applied, the FastMap technique tries to maintain the distances among the objects as close as they are in the original space. In general it is not possible, so, the FastMap minimizes the distortion through distributing the distance errors among the target dimensions.

The distribution of the error is performed using the following stress function:

$$
\text { stress }=\sqrt{\frac{\sum_{i, j}\left(\hat{d}_{i, j}-d_{i, j}\right)^{2}}{\sum_{i, j} d_{i, j}^{2}}} .
$$

where, $d_{i, j}$ is the distance between object $O_{i}$ and object $O_{j}$ and $\hat{d}_{i, j}$ is the distance between their mapped counterparts. In short, the stress function gives the relative error that the distances in the $k$-dimensional space suffer, on the average.

Based on the FastMap algorithm, we developed a tool named FastMapDB, whose main interaction window is presented in Figure 1. This tool works by reducing the dimensionality of any multidimensional data so that the resulting dimensionality is always three. Therefore, the mapped data can be plotted inside a tridimensional Euclidean space where the user is able to interactively and visually exploit them. Once the data items are plotted in the tridimensional space, it becomes possible to observe the similarity between the data elements, i.e., similar data should be closely plotted and different items should be far away from each other. Such representation is quite natural and intuitive to any user.

\section{Extractors to be compared}

The aim of the image feature extractors is to capture the essential characteristics, or properties, of the images by reducing their complexity while retaining as many salient details as possible, allowing to build image mining systems that employ CBIR techniques. In this work, we develop a technique to perform a comparative analysis of feature extractor algorithms. For illustration purposes, we have chosen a set of four extractors, previously proposed in the literature.

The first chosen extractor uses Gabor functions [11, 12], which is based on texture measurements to represent images through their rugosity. This extractor allows to build a feature vector computing the mean $\left(\mu_{s k}\right)$ and standard deviation $\left(\sigma_{s k}\right)$ for $S$ scales and $K$ orientation axes generated over the image by the Gabor functions. For example, for two scales $(S=2)$ and six orientation axes $(K=6)$, the resulting feature vector is composed of 24 values $(2 * 6$ means and $2 * 6$ standard deviations). The other three extractors employ Daubechies wavelets [18]. The Daubechies wavelets are normally used to extract features based on the color 
distribution over the scaled sub-spaces, applying three statistical measurements. The size of the feature vectors is proportional to the number of levels used in the multiresolution process. Hence, given an image $I(x, y)$, with $M x N$ pixels with depth $P$ and its histogram $H(P)$, we compute the features over each sub-space as

$$
\text { Mean }=\frac{1}{M \cdot N} \sum_{i=1}^{N} \sum_{j=1}^{M} I(i, j)
$$$$
\text { Entropy }=-\sum_{i=1}^{P} H(I) \cdot \log _{2}(i) \text { and Energy }=\sum_{i=1}^{P}[H(i)]^{2}
$$

Therefore, it is possible to extract features for evaluating the average brightness (given by the mean), the smoothness (given by the entropy), and the uniformity (given by the energy) of the images.

\section{The proposed metric-based framework}

The usual procedure to evaluate the discrimination power of a feature extractor is to compare, for each retrieval query, the retrieval results and the set of results expected by a specialist, plotting precision and recall curves. However, preparing such graphs is a time-consuming task which demands intense support of a specialist to evaluate the results of each query performed. In this paper we propose the use of two spatial measurements as a first evaluation technique to verify the applicability of a new extractor, before spending more time and effort with the traditional precision and recall approach, so only extractor algorithms showing good potential would be further analyzed.

\subsection{Basic properties of an image dataset}

In this paper, we name a group of images considered similar as a "class of similar images". For each class we define two properties: condensation and detachment. Ideally, a class of images should have its elements condensed, i.e., the class should originate a clearly bounded region in the mapped space. Different classes should occupy separated regions, so the condensed spatial region of one class should not be overlapped by elements of another class. Therefore, each class should be detached from each other, in some degree. These two properties are the base of our methodology.

As both the condensation and the detachment properties are obtained in a tridimensional mapped space, both spatial feature vectors (those where the number of features is constant for every image) and metric-based feature vectors (those where the number of features can vary freely, but follows a metric distance function) can be evaluated.
Therefore, not just the feature vectors, but also the distance functions can be evaluated.

\subsection{Feature measurements}

The first measurement we propose is based on the detachment property, and is named ClassClassifier. For a given class, the ClassClassifier states whether or not a given class is detached from the other classes. The second proposed measurement, based on the condensation property, is named ClassVariance. It states how condensed is each class. It corresponds to the distances' variance of the elements to the center of the class.

These measures are not easily appreciated in a given dataset due to the high dimensional nature of the data under analysis. Therefore, to corroborate our ideas, in the experiments section we promote the visualization of the classes in the test dataset in a tridimensional space.

\subsubsection{The ClassClassifier measurement}

The ClassClassifier of a given class $C$ can be thought of as how detached a class is from the others. To determine this detachment, it is necessary to identify the region occupied by class $C$ regarding the complete test dataset. We assume that this region is the Minimum Bounding Sphere (MBS) within which every element of the class is contained.

To determine the $M B S$ of a class, it is necessary to identify its most central element $\left(\right.$ mce $\left._{c}\right)$, which is the element closest to the center of the class's MBS. It can be found as follows. Given a distance function $d: \mathscr{D} x \mathscr{D} \rightarrow \mathbb{R}^{+}$, for every element $s_{i} \in S_{C}$, where $S_{c}$ is the set of images of the class $C$ in the test dataset $S$, calculate

$$
\sum_{s_{j} \in S_{c}} d\left(s_{i}, s_{j}\right)
$$

The element mce $_{c}$ is the element $s_{i}$ that minimizes the equation 4 . Then, the radius of the class $C$ is given by

$$
\max \left(d\left(m c e_{c}, s_{j}\right)\right), s_{j} \in S_{c} .
$$

Once identified the $m c e_{c}$ and the radius of the given class $C$, it is required to evaluate how much the other classes invade the $M B S$ of each class $C$. This can be performed measuring the distance from the mce $_{c}$ to every element of the other classes. We consider that the elements whose distance from $m_{c} e_{c}$ is smaller or equal to the radius of the class $C$ invade the MBS of class $C$. For a given class, the ClassClassifier measurement assumes 1 (fully detached) if the class is not invaded by other classes' elements, or 0 otherwise. The experiments showed that the proposed measurement indicates a misconception of images by the feature extractors. Yet, it can be calculated with linear 
computational cost regarding the number of elements and the number of classes in the dataset.

\subsubsection{The Class Variance measurement}

The ClassVariance of a given class $C$ can be thought of as the spreading of the elements of this class around the center of the $n$-dimensional sub-space defined by its elements. Knowing the $m c e_{c}$ and the radius of class $C$, we can calculate the ClassVariance. The average distance of the elements of the class $C$ to the $m c e_{c}$ is calculated as

$$
\bar{d}=\frac{\sum_{s_{j} \in S_{c}} d\left(\text { mce }_{c}, s_{j}\right)}{n-1}, s_{j} \neq m c e_{c} .
$$

where $n$ is the cardinality of class $C$, and the variance of the class is given by

$$
s^{2}=\frac{\sum_{s_{j} \in S_{c}}\left(d\left(m c e_{c}, s_{j}\right)-\bar{d}\right)}{n-1}, s_{j} \in m c e_{c} .
$$

The variance of the class indicates how condensed the class is and, consequently, the degree of similarity that the feature extractor is able to devote to the corresponding images.

\subsection{The spatial visualization}

We take advantage of interactive visualization mechanisms to corroborate the results obtained by the feature extractors measurements. This procedure is adequate since our objective is to evaluate how much a feature vector/distance function captures the human perceptual similarity notion. The measurements we developed are tightly related to the spatial notion.

As previously described, the FastMapDB tool provides the mechanism to visually present multidimensional items and classify them. Thus, to evaluate a new extractor, the feature vectors of the test image database are processed by the FastMap algorithm and plotted by the FastMapDB tool, the elements' shape and color are used to distinguish their classes. Our visual approach is based on two conjectures:

1) the numeric features extracted from a given image can represent the visual characteristics of the image as perceived by humans. The accuracy of this representation depends on the extractor used; and

2) visualization techniques can be efficiently employed to analyze data that, in pure format, is meaningless or, otherwise, too hard to figure out.

These conjectures led us to conclude that the visualization of feature vectors could reveal their behavior, i.e., their accuracy in characterizing the images whose features were extracted. Also, since the visualization can provide a better comprehension of our approach, we use this resource during the experiments.

Clusters in the visualization of the extracted features of the test dataset indicate condensed classes. A clear separation between the classes indicates distinct (detached) classes, as well as an intuitive perception of how different, or apart, they are, and what pairs of classes are detached and what are not.

\subsection{Data requirements}

To apply our procedure, it is necessary to use controlled data sets. That is, the test data set must be composed of a limited number of well-characterized classes of images. So the extractors can be evaluated to see if they maintain the image classes through their feature vectors.

The classes of images of the test database must present evident and explicit inner similarity, with reduced cross similarity between classes. Therefore, after assuring the characterization of each of the classes of images, the researcher can benefit from the similarity/dissimilarity between the diversity of classes to evaluate and fine-tune the feature extractor being evaluated. The researcher can define what kinds of discrimination tests are to be performed in function of a guided set of classes and, so, develop a gradual comprehension of the extractor being developed through a known similarity/dissimilarity environment.

The benefits of this procedure include the fact that the tests can be executed faster without the constant participation of a specialist in the image domain, as the specialist characterization database is necessary just once to build a test platform. Also, our proposed approach provides a precise and comprehensive evaluation of the feature extractor, what can be used as a first step to evaluate features extraction algorithms.

\section{Experiments}

To evaluate the proposed idea, we conducted a set of experiments performed over a test image data set, having their features generated by four wavelet transforms, as explained in Section 3. The experiments were performed in two phases. In the first one we executed $k$-nearest-neighbor queries on the features from the test image data set to obtain the precision and recall measurements. In the second phase, we applied the approach described in this paper.

\subsection{Experimental data}

We evaluated the proposed idea using both synthetic and real test data sets. However, due to space limitations, here we only present the results performed on a representative set of 500 real medical images. This set includes 7 well-defined classes with 30 similar images each, totaling 210 images. 
The medical descriptions of these 7 classes are: sagital central human brain, abdominal, axial pelvis, axial human brain, angiographic, sagital spine and border human brain sagital, respectively, as can be seen in figure 2 .


- (a) sagital central human brain

- (b) abdominal

- (c) axial pelvis

-(d) axial human brain

-(e) angiographic

- (f) sagital spine

(g) sagital border human brain

Figure 2. Representative sample images of the seven classes used in the experiments and the colors assigned to each class in the visualization

The remaining 290 images in the data set are images completely distinct from those seven classes, so they have no inner common characteristics with the classified images. These images are used as noisy data, to allow performing a full and robust precision and recall procedure. This ensemble fulfills the test dataset requirements to fully evaluate our proposed technique.

\subsection{Test feature vectors}

Four sets of features were computed from the image data set, namely, DMean, DEnergy, DEntropy and Gabor. The first three ones are the mean, energy and entropy of the nine higher-frequency orthogonal sub-spaces generated by the Daubechies extractor; the fourth one was generated by the Gabor extractor, as described in section 3.

\subsection{Objectives of the experiments}

The goal of the experiments is to verify if the proposed methodology is able to answer the four objectives stated in Section 1. A representative image from each of the seven classes that the extractors are supposed to characterize can be observed in figure 2 . The characterization shall be perceived through the use of the feature vectors to answer similarity queries. Hence, the experiments aim to demonstrate the effectiveness of the proposed technique to compare the relative quality of the four extractors. The quality is measured first by the traditional precision and recall plots, and then by the proposed measurements. The results are compared.

\subsection{Precision and recall evaluation}

The precision and recall measurements were performed using $k$-nearest-neighbor similarity queries over the image database. The images were retrieved based on their feature vectors using the Euclidean distance function as the dissimilarity function.

\subsubsection{Precision and recall setup.}

We performed five queries for each of the seven image classes and plotted the average value found. In each query, a random image of a given class was used. Each query was expected to sort the 500 stored images regarding their similarity to the query image. Therefore, a total of 35 queries were executed for each of the four extractors under analysis.

The ideal extractor should retrieve the 29 remaining elements of the same class of the query center (the query image). And, the precision and recall technique intends to measure how far from this idealistic scenario a given extractor is. Thus, a sequence of experiments for the different extractors must show their relative efficiency.

\subsubsection{Precision and recall results.}

The precision and recall plots for the four extractors are presented in Figure 3, where a recall value of $100 \%$ corresponds to asking for 29 nearest neighbors. The plots show that the DEntropy extractor provides the better retrieval

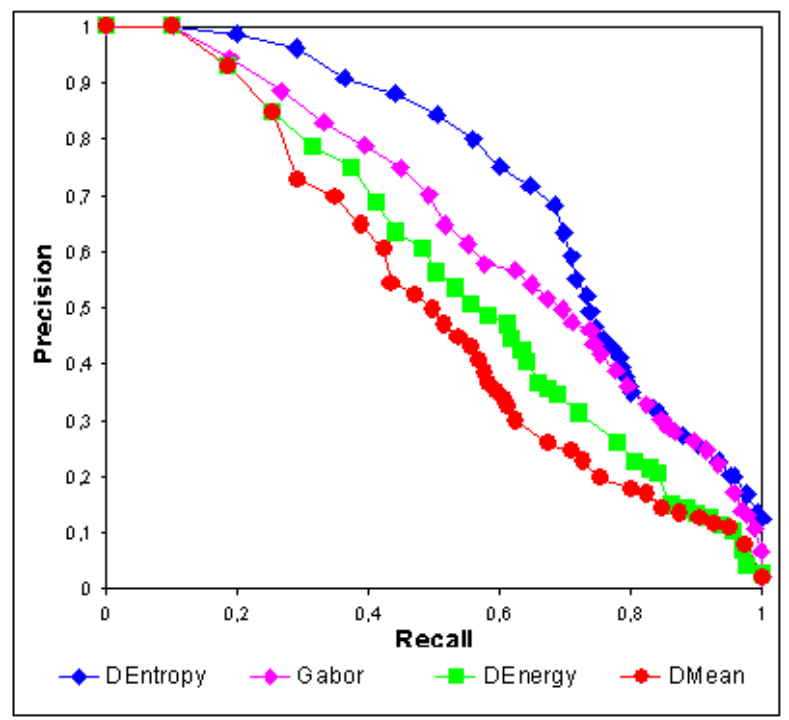

Figure 3. The precision and recall graphs computed for the four feature extractors 
ability among the four extractors. Following, in order of retrieval ability, according to the graph, were the Gabor, the DEnergy and the DMean extractor.

The use of the precision and recall graphs required a massive effort of a specialist to validate the answers for each of the 140 queries ( 35 for each of the four extractors) in an environment with 500 images.

\subsection{Metrical evaluation}

Our proposed measurements ClassClassifier and ClassVariance are automatically computed. The results obtained for each one of the 7 classes and for each one of the 4 extractors are presented in Table 1 .

The table shows that the DEntropy was the feature extractor that best categorized the image classes with the ClassClassifier pointing that 6 out of 7 classes were fully detached from the others. Meanwhile, the DMean extractor could only separate 3 out of the 7 classes. Their ClassVariance measurement also supports this result, as the more spread the class is, the larger are the chances that it will invade another class's space. The DEntropy extractor presents a ClassVariance of 1.93, and the DMean extractor presents a more confusing indicator, 3.33.

Looking at the Gabor and at the DEnergy measurements, it can be seen that the former presents worse condensation than the DEntropy, and that the later presents a slightly better result compared to the DMean features set. This overall analysis is also confirmed by the precision and recall measurements (Figure 3 ).

Table 1. Class Variance and ClassClassifiermeasurements for the seven image groups, calculated for each of the four feature extractors under analysis

\begin{tabular}{|l|r|r|r|r|r|r|r|r|}
\hline $\begin{array}{l}\text { Extractor } \\
\text { Class }\end{array}$ & \multicolumn{2}{|c|}{ DEntropy } & \multicolumn{2}{|c|}{ Gabor } & \multicolumn{2}{|c|}{ DEnergy } & \multicolumn{2}{|c|}{ DMean } \\
\hline $\mathbf{0}$ & 1.00 & 1 & 0.63 & 0 & 0.41 & 0 & 1.00 & 1 \\
\hline $\mathbf{1}$ & 0.21 & 1 & 1.00 & 1 & 1.00 & 1 & 0.40 & 0 \\
\hline $\mathbf{2}$ & 0.07 & 1 & 0.24 & 1 & 0.20 & 1 & 0.46 & 0 \\
\hline $\mathbf{3}$ & 0.33 & 0 & 0.44 & 1 & 0.10 & 1 & 0.65 & 0 \\
\hline $\mathbf{4}$ & 0.15 & 1 & 0.17 & 1 & 0.29 & 0 & 0.12 & 1 \\
\hline $\mathbf{5}$ & 0.17 & 1 & 0.06 & 1 & 0.08 & 0 & 0.41 & 0 \\
\hline $\mathbf{6}$ & 0.11 & 1 & 0.31 & 1 & 0.63 & 0 & 0.29 & 1 \\
\hline Sum & $\mathbf{1 . 9 3}$ & $\mathbf{6}$ & $\mathbf{2 . 8 4}$ & $\mathbf{6}$ & $\mathbf{2 . 7 2}$ & $\mathbf{3}$ & $\mathbf{3 . 3 3}$ & $\mathbf{3}$ \\
\hline
\end{tabular}

\subsection{Spatial visualization}

Figure 4 shows the visualizations produced by the FastMapDB tool. Looking at these visualizations, it can be seen that the separation of the clusters is more evident when the features are generated by the DEntropy extractor. The visualization also points out that the Gabor extractor (Figure 4(b)) produces clusters slightly less condensed than the DEntropy extractor. The remaining extractors DEnergy and DMean were not able to satisfactorily separate the clusters, although they presented a reasonable condensation power, as is shown in Figures 4(c) and 4(d).

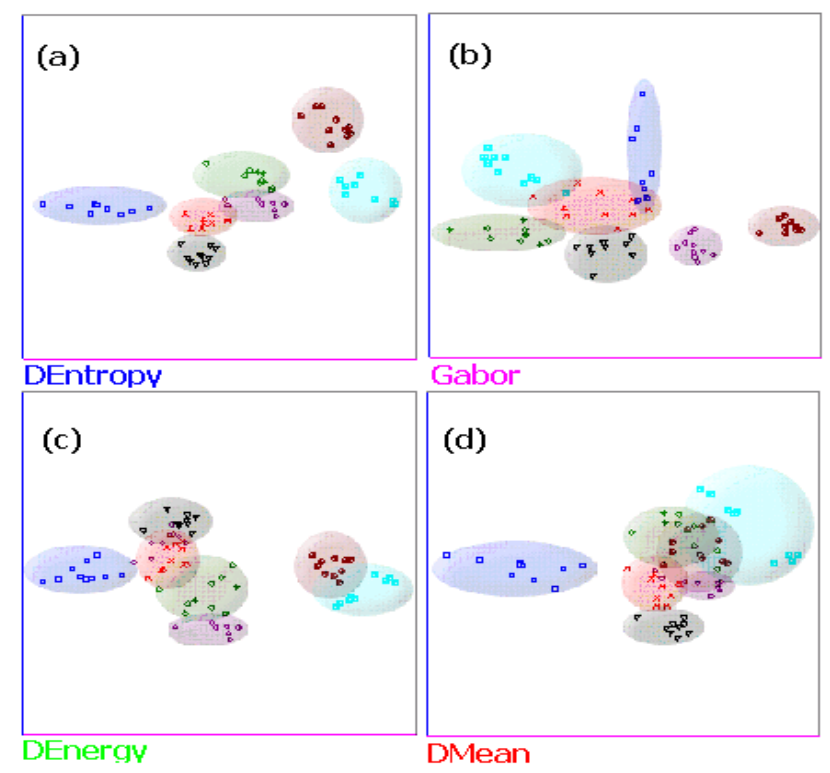

Figure 4. 2D projection of the four sets of feature vectors mapped to the 3D space. Depth cannot be perceived

The overall visualization meets the measurements presented in the table 1 . Therefore, the numerical values of the table summarize the same results observed in the visualization.

As one can see, the visualization tool gives an insight of the extractor algorithm ability for image characterization and consequently for image retrieval.

\section{Conclusions}

In this paper we presented a powerful and easy-to-use technique to evaluate the usefulness of image feature extractor algorithms for image mining, allowing its use in content-based image retrieval applications. The technique is based on two measurements that embody spatial properties naturally perceived in spatial domains, and that resembles the human perception of similarity in image sets. The results of our experiments showed that the proposed technique was able to highlight the relative efficiency of the four different feature extractors, in accordance with the more complex precision and recall analysis.

It is important to emphasize that our technique is not intended to substitute the precision and recall methodology but to help the researcher to better understand and refine a feature extractor being developed in a timely manner. The proposed method does not demand large data sets where it can be evaluated, but rather a well-known one, that includes 
subsets of representative images that correspond to the classes of images expected in the image domain.

The method can be used to quickly evaluate the behavior of a given extractor, rather than to validate it. Therefore, it satisfies the objectives of this paper as it enables answering whether a feature extractor is adequate to discriminate images in a given domain and, whenever there is more than one extractor, it chooses which is the better to classify images in general and which is better to classify images for a given class. It also provides clues to the feature extractors/distance functions developer, so it can improve/fine tune the algorithms. Therefore it can be used as a first step in the analysis process of evaluating the applicability of image features extraction algorithms, before to spend a large deal of time and effort to evaluate such algorithms by the traditional precision and recall.

\section{Acknowledgments}

The authors are grateful to the Center of Image Science of the Clinical Hospital of the University of São Paulo at Ribeirão Preto - Brazil, which kindly provided the project with the images used in the experiments. This research has been supported, in part, by the Sao Paulo State Research Foundation (FAPESP) under grants 03/01144-4 and 02/07318-1, and by the Brazilian National Research Council (CNPq) under grants 52.1685/98-6, 860.068/00-7 and 52.1267/96-0.

\section{References}

[1] M. G. Albanesi, S. Bandelli, and M. Ferretti, "Quantitative assessment of qualitative color perception in image database retrieval," Proc. 11th Intl. Conference on Image Analysis and Processing, Thessaloniki, Greece, October 7-10, 2001, pp. 410-415.

[2] E. Albuz, E. Kocalar, and A. A. Khokhar, "Scalable Color Image Indexing and Retrieval Using Vector Wavelets," IEEE Transactions on Knowledge and Data Engineering (TKDE), Vol. 13, No. 5, September/October 2001, pp. 851-861.

[3] R. Baeza-Yates and B. A. Ribeiro-Neto, Modern Information Retrieval. Wokingham, UK: Addison-Wesley, 1999.

[4] R. A. Becker, S. G. Eick, and G. J. Wills, "Visualizing Network Data," IEEE Transactions on Visualization and Computer Graphics, Vol. 1, No. 1, 1995, pp. 16-28.

[5] C. Faloutsos and K.-I. D. Lin, "FastMap: A Fast Algorithm for Indexing, Data-Mining and Visualization of Traditional and Multimedia Datasets," Proc. ACM SIGMOD International Conference on Management of Data, San Jose, CA, 1995, pp. 163-174.

[6] Y.-H. Fua, M. O. Ward, and A. Rundensteiner, "Hierarchical Parallel Coordinates for Exploration of Large Datasets,"
Proc. IEEE Visualization'99

[7] A. Inselberg and B. Dimsdale, "Parallel Coordinates: A Tool for Visualizing Multidimensional Geometry," Proc. IEEE Visualization, 1990, pp. 361-370.

[8] E. Kandogan, "Visualizing Multi-dimensional Clusters, Trends, and Outliers using Star Coordinates," Proc. 7th ACM SIGKDD International Conference on Knowledge Discovery and Data Mining, San Francisco, CA, USA, pp. 107-116.

[9] D. A. Keim, "Designing Pixel-Oriented Visualization Techniques: Theory and Applications," IEEE Transactions on Visualization and Computer Graphics, Vol. 6, No. 1, January-March 2000, pp. 59-78.

[10] D. A. Keim, "Information Visualization and Visual Data Mining," IEEE Transactions on Visualization and Computer Graphics, Vol. 8, No. 1, January-March 2002, pp. 1-8.

[11] T. S. Lee, "Image Representation Using 2D Gabor Wavelets," IEEE Transactions on Pattern Analysis and Machine Intelligence, Vol. 18, No. 10

[12] B. S. Manjunath and W. Y. Ma, "Texture Features for Browsing and Retrieval of Image Data," IEEE Transactions on Pattern Analysis and Machine Intelligence, Vol. 18, No. 8, August 1996, pp. 837-842.

[13] M. Mirmehdi and R. Perissamy, "Perceptual Image Indexing and Retrieval," Journal of Visual Communication and Image Representation, Vol. 13, No. 4, December 2002, pp. 460475.

[14] H. Müller, N. Michoux, D. Bandon, and A. Geissbuhler, "A review of content-based image retrieval systems in medical applications-clinical benefits and future directions," International Journal of Medical Informatics, Vol. 73, No. 1, February 2004, pp. 1-23.

[15] Y. Rubner and C. Tomasi, Perceptual Metrics for Image Database Navigation: Kluwer Academic Publishers, 2001.

[16] Y. Rui, T. S. Huang, and S.-F. Chang, "Image retrieval: current techniques, promising directions and open issues," Journal of Visual Communication and Image Representation, Vol. 10, No. 1, April 1999, pp. 39-62.

[17] A. W. M. Smeulders, M. Worring, S. Santini, A. Gupta, and R. Jain, "Content-Based Image Retrieval at the End of the Early Years," IEEE Transactions on Pattern Analysis and Machine Intelligence, Vol. 22, No. 12, December 2000, pp. 1349-1380.

[18] E. J. Stollnitz, T. D. DeRose, and D. H. Salesin, Wavelets for Computer Graphics - Theory and Applications. San Francisco, CA: Morgan Kaufmann Publishers, Inc, 1996.

[19] W. S. Torgenson, "Multidimensional Scaling: I. Theory and Methods," vol. 17: Psychometrika, 1952, pp. 401-419.

[20] A. Vailaya, M. A. T. Figueiredo, A. K. Jain, and H.-J. Zhang, "Image classification for content-based indexing," IEEE Transactions on Image Processing, Vol. 10, No. 1, January 2001, pp. 117-30.

[21] C. Ware, Information Visualization: Perception for design: Morgan Kaufmann Publishers, 2000. 